(1)

CrossMark

\title{
Introduction to precision medicine in COPD
}

\author{
Janice M. Leung ${ }^{1,2}$, Ma'en Obeidat ${ }^{1,2}$, Mohsen Sadatsafavi ${ }^{3}$ and Don D. $\operatorname{Sin}^{1,2}$ \\ Number 4 in the series \\ "Controversies in COPD: What Can be Done to Move the Field Forward?" \\ Edited by D.D. Sin
}

\begin{abstract}
Affiliations: 'UBC Centre for Heart Lung Innovation, University of British Columbia and St. Paul's Hospital, Vancouver, BC, Canada. ${ }^{2}$ Dept of Medicine, University of British Columbia and St. Paul's Hospital, Vancouver, BC, Canada. ${ }^{3}$ Faculty of Pharmaceutical Sciences, University of British Columbia, Vancouver, BC, Canada.
\end{abstract}

Correspondence: Don D. Sin, Room 385, Burrard Building, St. Paul's Hospital, 1081 Burrard Street, Vancouver, BC, Canada, V6Z 1Y6. E-mail: don.sinQhli.ubc.ca

@ERSpublications

COPD care needs to take into account individual variation in genes, environment and lifestyle. This review outlines the steps required for COPD care to move out of its "one-size-fits-all" approach to precision medicine. http://ow.ly/9UI830nlNb8

Cite this article as: Leung JM, Obeidat M, Sadatsafavi $\mathrm{M}$, et al. Introduction to precision medicine in COPD. Eur Respir J 2019; 53: 1802460 [https://doi.org/10.1183/13993003.02460-2018].

ABSTRACT Although there has been tremendous growth in our understanding of chronic obstructive pulmonary disease (COPD) and its pathophysiology over the past few decades, the pace of therapeutic innovation has been extremely slow. COPD is now widely accepted as a heterogeneous condition with multiple phenotypes and endotypes. Thus, there is a pressing need for COPD care to move from the current "one-size-fits-all" approach to a precision medicine approach that takes into account individual patient variability in genes, environment and lifestyle. Precision medicine is enabled by biomarkers that can: 1) accurately identify subgroups of patients who are most likely to benefit from therapeutics and those who will only experience harm (predictive biomarkers); 2) predict therapeutic responses to drugs at an individual level (response biomarkers); and 3) segregate patients who are at risk of poor outcomes from those who have relatively stable disease (prognostic biomarkers). In this essay, we will discuss the current concept of precision medicine and its relevance for COPD and explore ways to implement precision medicine for millions of patients across the world with COPD.

No. 1: Kim V, Aaron SD. What is a COPD exacerbation? Current definitions, pitfalls, challenges and opportunities for improvement. Eur Respir J 2018; 52: 1801261. No. 2: Washko GR, Parraga G. COPD biomarkers and phenotypes: opportunities for better outcomes with precision imaging. Eur Respir J 2018; 52: 1801570. No. 3: Soriano JB, Polverino F, Cosio BG. What is early COPD and why is it important? Eur Respir J 2018; 52: 1801448.

Received: Dec 292018 | Accepted after revision: Jan 122019

Copyright OERS 2019 


\section{Introduction}

Chronic obstructive pulmonary disease (COPD) is a major global burden, affecting $>300$ million people worldwide and accounting for 2.9 million deaths annually [1]. By 2040, this number will increase by $32 \%$ to 4.4 million deaths annually, making it the fourth leading cause of mortality (currently ninth), trailing only ischaemic heart disease, stroke and pneumonia [1]. This increase will be driven largely by the ageing populations around the world, decreasing mortality rates of competing causes of death (e.g. ischaemic heart disease), increased exposure to ambient indoor and outdoor pollution, and poor lifestyle choices including tobacco smoking. Although smoking rates have fallen dramatically in western countries, there are still 942 million men and 175 million women in the world who are current smokers [2]. Moreover, according to the World Health Organization (WHO), there are 4.2 million deaths per year attributed to ambient air pollution and 3.8 million deaths per year from biomass exposure from dirty stoves and fuels. Remarkably, WHO estimates that $91 \%$ of the world's population lives in areas where air quality is below the lower limit of acceptability (for health) [3]. The dramatic rise in the use of e-cigarettes [4] and smoked marijuana in the USA and elsewhere poses additional risks that to date have not been well quantified [5].

While there has been an explosion in scientific interest and publications on COPD over the past 20 years (figure 1), there has been little progress in the number of new therapies for this condition. Indeed, over the past 30 years only one new therapeutic class has been successfully introduced to treat COPD patients (i.e. phosphodiesterase-4 (PDE4) inhibitors) [6]. The current pharmacologic therapies are bronchodilators (targeting $\beta 2$ adrenergic or muscarinic receptors in airway smooth cells) and corticosteroids [7]. Although on average these therapies reduce symptoms and modify risk of exacerbations, their impact on individual patients has not been fully evaluated. Most of the evidence for these therapies has been derived from large therapeutic trials in which the approach has been "one size fits all". In this essay, we will provide an overview of precision medicine in COPD and its prospects in changing the therapeutic landscape in COPD over the next decade.

\section{Precision medicine versus "evidence-based" care}

Currently, clinicians are encouraged to provide "evidence-based" care for their patients, which was originally defined as "the conscientious, explicit and judicious use of current best evidence in making decisions about the care of individual patients" [8]. The highest level of evidence is believed to be derived from multiple large randomised controlled trials (RCTs) that generate narrow confidence intervals [9]. Accordingly, over the past two decades there has been a rise in very large RCTs in COPD that have enrolled thousands (and in some cases tens of thousands) of patients to generate p-values (on primary outcomes) that fall below the "magic" threshold of 0.05 (two-tailed). Thus, a "positive" study is one that meets this threshold, whereas those that do not are considered "failures".

An obvious weakness of this evidence-based approach is that these RCTs generally do not consider the heterogeneity of disease or patients. There is mounting evidence that COPD is not a single disease entity but rather a collection of abnormalities, driven by different molecular processes or pathophysiologies [10]. Thus, for practicing clinicians, implementing evidence-based data from large therapeutic trials for individual patients in their everyday practice is an enormous challenge. Use of inhaled corticosteroids (ICS) is a prime example of this conundrum. Whereas evidence-based data would suggest that most COPD patients do not benefit from ICS therapy, clinicians in "real-world" practice often use ICS therapy for their patients with COPD. One important reason is that $\sim 10-25 \%$ of patients with COPD in the real

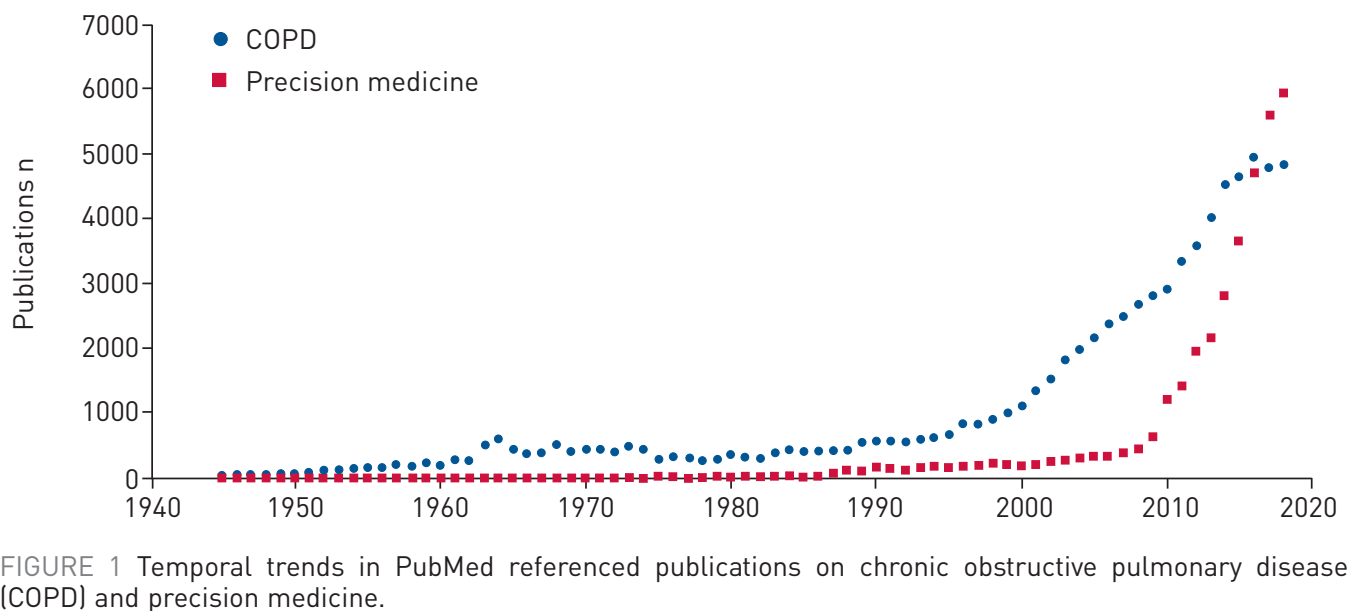


world also have asthma [11]. Although these patients are generally excluded in therapeutic RCTs, they cannot be ignored in clinical practice.

The weakness of the one-size-fits-all care approach has given rise to the concept of precision medicine [12]. Although there is no universally accepted definition of precision medicine, the National Institutes of Health (NIH) defines it as "treatments targeted to the needs of individual patients on the basis of genetic, biomarker, phenotypic, or psychosocial characteristics that distinguish a given patient from other patients with similar clinical presentations" [12] (box 1). Implicit in this definition is the goal of improving clinical outcomes for individual patients and minimising unnecessary side effects for those less likely to have a response to a particular treatment [13]. Simply, precision medicine is "prevention and treatment strategies that take into account individual variability in genes, environment and lifestyle" [12]. An extreme version of precision medicine is personalised medicine, in which the entire management is structured for (or catered to) an individual and thus may not be generalisable beyond that patient.

\section{Biomarkers: an essential component for precision medicine}

One key component of enabling precision medicine at the bedside is biomarkers. Although many consider biomarkers synonymous with "blood tests", an NIH expert panel defined them more broadly "as factors that are objectively measured and evaluated as indicators of normal biological or pathological processes, or pharmacological responses to therapeutic intervention" [14]. In practice, biomarkers can be sputum or blood tests, imaging modalities, prediction rules or, more broadly, treatable traits [15]. Biomarkers can be sub-classified as diagnostic, response (further divided into pharmacodynamic biomarkers and surrogate endpoint biomarkers), prognostic (correlated with clinical outcome but not necessarily directly related to specific mechanisms) or predictive (predict response to specific targeted drug interventions) [16].

The ratio of the forced expiratory volume in $1 \mathrm{~s}$ (FEV1) to the forced vital capacity, based on post-bronchodilator spirometry, is an example of a diagnostic biomarker. The body mass index, airflow obstruction, dyspnoea and exercise (BODE) index is an example of a prognostic biomarker, and provides information on the future risk of mortality in a given patient. Blood eosinophil count is an example of a predictive biomarker in determining therapeutic guidance for ICS. Serum concentration of $\alpha 1$-antitrypsin in those with emphysema related to SERPINA1 deficiency is an example of a response biomarker.

In the context of precision medicine, predictive biomarkers are of prime importance because they guide therapeutic choices and make therapies safer and more cost-effective. There are several biomarkers in practice that are commonly used to target specific therapies for specific subgroups of COPD patients (figure 2). For example, according to the National Emphysema Treatment Trial (NETT) study, the overall number of patients needed to treat (NNT) for lung volume reduction surgery (LVRS) to prevent one death

\section{BOX 1 Definitions}

Biomarkers: factors that are objectively measured and evaluated as indicators of normal biological or pathological processes, or pharmacological responses to therapeutic intervention.

Chronic obstructive pulmonary disease (COPD): a collection of disease entities characterised by symptoms of cough, shortness of breath and/ or sputum production and by persistent airflow limitation on spirometry in the absence of other major lung conditions such as bronchiectasis and asthma, and associated with at least 10 pack-years of smoking history or equivalent biomass exposure.

Endotype: subtype of a condition, which is defined by a distinct functional or pathobiological mechanism.

Genome-wide association studies (GWASs): a hypothesis-free approach to identify associations between genetic variants across the whole genome (locil and traits such as disease, quantitative traits and drug response.

$\mathbf{N}$ of 1 trial: a randomised controlled crossover trial in a single patient designed to establish optimal treatment for that patient.

Number needed to treat (NNT): the average number of patients who needed to be treated with the therapy in question to prevent at least one additional negative outcome.

Number needed to harm (NNH): the average number of patients who are treated with the therapy in question before at least one patient experiences a negative reaction to the therapy.

Phenotype: a set of observable characteristics in an individual resulting from the interaction of their genotype with the environment.

Precision medicine: an approach for disease treatment and prevention that takes into account individual variability in genes, environment and lifestyle for each person. In the context of COPD care, a satisfactory application of precision health is to find subgroups in which the NNT is $\leqslant 10$.

Predictive biomarker: a biomarker that can be used to predict therapeutic responses (beneficial or negative) in a given individual patient, enabling targeting of therapeutics to specific individuals.

Prognostic biomarker: a biomarker that can be used to accurately determine risk of important clinical events in the future such as disease progression, acute exacerbations, hospitalisations and mortality of COPD patients.

Response biomarker: a biomarker whose level changes in response to an exposure to a therapeutic product; can be subdivided into pharmacodynamic biomarkers, which are used to gauge biological effects of therapy, and surrogate endpoint biomarkers, which are used to estimate potential clinical impact of therapies. 


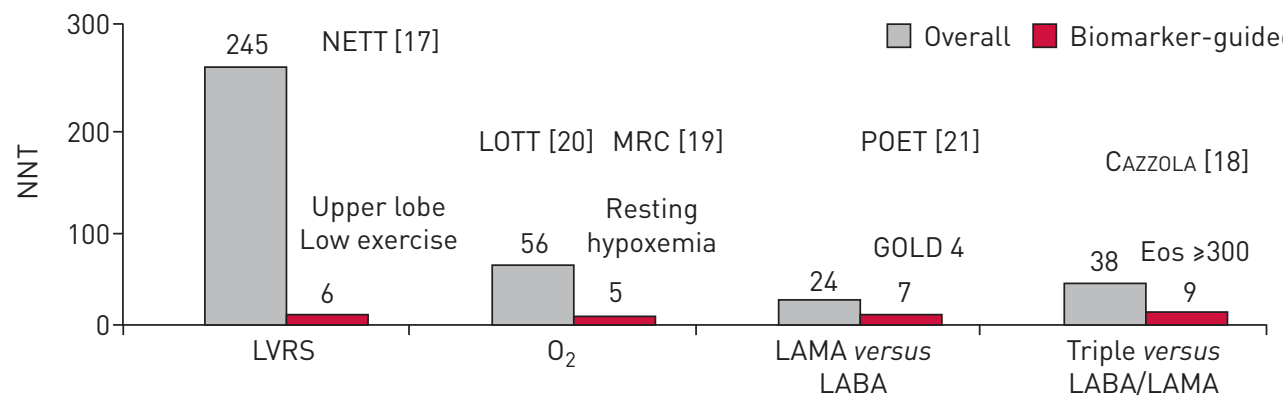
Outcomes: Prevent one death
Prevent one death
over 3 years
Prevent at least one exacerbation
over 5 years
over 1 year

FIGURE 2 A comparison of number needed to treat (NNT) between non-biomarker and biomarker-based approaches to chronic obstructive pulmonary disease (COPD) therapies currently available in most jurisdictions. x-axis denotes therapies currently available for COPD patients, with the therapeutic trial or meta-analysis from which NNTs were derived indicated above the bars. $y$-axis denotes NNT to prevent one event of clinical importance (over a fixed period of time) for each intervention with or without the use of biomarker(s). LVRS: lung volume reduction surgery; $\mathrm{O}_{2}$ : supplemental (domiciliary) oxygen therapy; LAMA: long-acting muscarinic antagonist; LABA: long-acting $\beta 2$ adrenergic agonist; Triple: a combination of inhaled corticosteroids and LABA and LAMA; NETT: National Emphysema Treatment Trial; LOTT: Long-term Oxygen Treatment Trial; MRC: Medical Research Council Oxygen Therapy Trial; POET: Prevention of Exacerbations with Tiotropium in COPD; GOLD: Global Initiative for Chronic Obstructive Lung Disease; Eos: blood eosinophil count in absolute scale (cells. $\mu \mathrm{L}^{-1}$ ). NNT is calculated based on persons.

over 5 years is 246 [17]. However, by targeting LVRS to those patients with predominantly upper lobe disease (as determined by thoracic computed tomography (CT)) and low exercise capacity (defined as a maximal workload of $25 \mathrm{~W}$ in women and $40 \mathrm{~W}$ in men post-rehabilitation), the NNT improves to seven [17]. Cazzola et al. [18] showed in their meta-analysis of RCTs that provision of "triple" therapy consisting of an ICS, a long-acting $\beta 2$ agonist (LABA) and a long-acting muscarinic antagonist (LAMA) was associated with an NNT of 38 to prevent at least one exacerbation over 1 year compared with dual LABA/LAMA combination therapy. However, this NNT improved to nine when triple therapy was given only to those with "high" blood eosinophils $\left(\geqslant 300\right.$ cells $\left.\mu \mathrm{L}^{-1}\right)$. Treatment with continuous oxygen therapy is associated with an NNT of five to prevent one death over 3 years in those with resting hypoxaemia (arterial oxygen tension $\left(\mathrm{PaO}_{2}\right)<60 \mathrm{mmHg}$ ) [19], whereas the NNT inflates to 56 for provision of continuous oxygen therapy for those with just nocturnal or exertional hypoxaemia [20]. In the Prevention of Exacerbations with Tiotropium in COPD (POET) trial, the use of a LABA (tiotropium) was associated with an $18 \%$ relative reduction in the risk of exacerbations compared with a LABA (salmeterol) in patients with moderate to severe COPD (corresponding to an NNT of 24 over 1 year). A subgroup analysis showed that in patients with a Global Initiative for Chronic Obstructive Lung Disease (GOLD) severity grade of 4 (FEV $1<30 \%$ of predicted) [7], there was a 36\% relative risk reduction (corresponding to an NNT of seven) with LAMA use [21]. More recently, use of bronchoscopy to ascertain collateral ventilation was demonstrated to be an effective biomarker in predicting therapeutic responses to endobronchial valve therapy in COPD patients with emphysema [22].

\section{Biomarkers as a gateway for future drug development}

Although there have been notable improvements in the care of COPD patients over the past several decades, the pace of new developments in respiratory diseases has been extremely slow and fraught with repeated failures. The cumulative probability of respiratory drugs reaching the clinic is only 3\% (from phase I to regulatory approval), whereas it is $14 \%$ for HIV/AIDS drugs and $7 \%$ for cancer therapeutics [6]. The greatest attrition occurs during phase II and III studies, mostly owing to lack of efficacy (accounting for $\sim 60 \%$ of failures) [6]. Traditionally, pharmaceutical companies have relied on preclinical animal models to assess potential therapeutic targets and compounds. However, because they are poorly predictive of the human condition, they have been largely abandoned in favour of genomics-based approaches to drug discovery and development in COPD [23].

There are several common reasons for the repeated failures of drug development in COPD (table 1), including inadequate target engagement of the drug, poor patient selection and use of clinical endpoints that are insensitive or inaccurate for detecting adequate treatment responses [24]. Drug companies have found that discovery and implementation of biomarkers to phenotype patients and select only those who are likely to experience benefit from the drug dramatically increases the probability of success of novel 
TABLE 1 Biomarkers as potential solutions to address common barriers in drug development programmes

Barriers Details $\quad$ Potential biomarker solutions

$\begin{array}{ll}\begin{array}{l}\text { Poor target engagement or } \\ \text { linkage to disease or disease }\end{array} & \begin{array}{l}\text { Compounds are targeted at molecules that are not } \\ \text { relevant in the pathogenesis of the human disease } \\ \text { phenotypes }\end{array}\end{array}$
phenotypes relevant in the pathogenesis of the human disease condition
Dose of drug limited by characteristics of compound or target tissue

Poor patient selection
Once in humans, because of safety concerns or undesirable pharmacokinetic properties, the dose cannot be optimised

COPD is a heterogeneous disease and as such unselected patients are likely to have variable responses to treatment, diluting the effects of the drug
- Linkage of target with human genetics data relevant for disease in question

- Perform GWAS, rare functional mutational, Mendelian randomisation or other biomarker analyses

- Pharmacodynamic biomarker to assess engagement of target molecule of interest

- Pharmacodynamic biomarkers to assess pharmacokinetic properties of compound

- Response biomarkers to assess potential efficacy or toxicity of drug

- Predictive biomarker to ensure that subpopulation selected will most likely respond to drug

GWAS: genome-wide association analysis; COPD: chronic obstructive pulmonary disease.

drugs in phase IIa trials from $\sim 29 \%$ (pre-biomarker implementation) to $82 \%$ (with biomarker implementation) [24].

Although it is widely acknowledged that biomarkers are urgently needed to address gaps in drug development, biomarker implementation has been difficult owing to multiple barriers (table 2). Academics often work in silos. However, as with therapeutic products, biomarker development requires academics to work closely with industrial partners to "push" discovery into validation and ultimately into clinical utility studies for regulatory qualification and clinical implementation. Because this process usually takes many years, often requiring millions of pounds in investment (with no guarantee of success), very few companies are willing to take these risks by themselves. To accelerate biomarker development, novel public-private funding models will be needed in the future to de-risk these efforts. Further, similar to genomics data, biomarker data should be publically available on searchable and easy-to-find databases with adequate annotations and on high-quality platforms to enable "data mining", reproduction and validation of results and to prevent unnecessary duplication of efforts.

\section{Other enablers of precision medicine}

Beyond biomarkers, clinicians may use clinical features to guide therapeutic choices. For instance, symptoms of chronic bronchitis (e.g. daily productive cough) significantly modify the therapeutic efficacy

TABLE 2 Common barriers to biomarker discovery and implementation and potential solutions

\section{Barriers}

Fractured developmental process because of inadequate collaboration between academia and industry [50]

Poor reproducibility of preclinical data

Poor stewardship and dissemination of original data for wider use
Details

Biomarker discovery occurs in academia, and regulatory approval and marketing of biomarkers require industry support

$>50 \%$ of preclinical biomarker data cannot be adequately replicated in subsequent studies [51]

After papers are published, the biomarker data are often not accessible by the scientific community
Potential solutions

- Involvement of industry as part of the discovery team

- Funding to encourage joint academicindustry partnerships

- Make biomarker data more widely available

- Use large samples from well-phenotyped patients

- Perform external replication during discovery

- Use standardised and reproducible platforms and analytical methods

- Original biomarker data should be findable, accessible, interoperable and reusable (FAIR) [52] 
of roflumilast, a PDE4 inhibitor [25]. These features are also being used to pursue investigational treatments in COPD, including mucolytics and radiofrequency as well as cryoablation therapies of the airways (clinicaltrials.gov). Pharmacogenomics, which combines pharmacology and genetics to study how genes affect patient responses to particular drugs, is another enabler of precision medicine. Although there are no clear examples of pharmacogenomics in COPD, in cystic fibrosis, genotyping (G551D) is currently being used to guide therapeutic choices for ivacaftor [13]. Given the heterogeneity of COPD, precision medicine would also be enabled by targeting therapies based on disease endotypes. For example, anti-IgE therapy may be highly effective in COPD patients whose disease is driven largely by a severe allergic endotype [26].

\section{Scale of estimation versus scale of interest in precision medicine}

In addition to the above, in precision medicine the benefits as well as the risks of therapeutic products need to be accurately assessed. Treatment effects in clinical trials and observation studies are often estimated and reported on a relative scale, such as per cent reduction in the risk or rate of events (scale of estimation). However, in precision medicine, what matters more is the absolute risk or rate that is modified by the therapy of interest (scale of interest). This approach lends itself to metrics that are easily understood in clinical practice and thus have clinical relevance: the "number needed to treat" (NNT) and the "number needed to harm" (NNH). The original concept of NNT (or NNH) is based on the following formula: 1 divided by the absolute risk reduction between the experimental therapy and placebo over the study's duration [27].

In most therapeutic trials in COPD, the primary outcomes are derived from a Cox (proportional hazard) survival model (to compare time to first exacerbation between treatment arms), logistic regression (to compare the proportion of patients with at least one exacerbation during follow-up between treatment arms) or count models such as Poisson or negative binomial regression (to compare the rate of exacerbations during follow-up between treatment arms). These regression models produce treatment effects, which are represented as hazard ratios, odds ratios or rate ratios, respectively. These parameters all generate numbers on a relative scale and not on an absolute scale, which can cause confusion for the reader.

An example to illustrate the major difference in risks between relative and absolute scales can be found in the Macrolide Azithromycin for Prevention of Exacerbations of COPD (MACRO) study. The MACRO study was a landmark clinical trial that demonstrated a $27 \%$ reduction in the risk of exacerbations with daily low-dose azithromycin therapy (rate ratio $0.73,95 \%$ CI $0.63-0.84, \mathrm{p}<0.001$ ) [28]. However, it is widely known that there is tremendous variability in the background rate of exacerbations across patients. A recent study [29] estimated that the range (containing 95\% of the study population) of annual background rate of moderate to severe exacerbations (without azithromycin therapy) was between 0.47 (i.e. one exacerbation every 564 days) and 4.22 (i.e. one exacerbation every 87 days). For the individual with a background exacerbation rate of 0.47 , a $27 \%$ relative reduction in the exacerbation rate with azithromycin would translate to a prevention of 0.13 exacerbations per year, corresponding to an event-based NNT of $1 / 0.127=7.88$. For those with a background exacerbation rate of 4.22 , azithromycin therapy would prevent 1.14 exacerbations per year, corresponding to an event-based NNT of 1/1.14=0.88. In this context, the provision of azithromycin is likely justifiable clinically and economically for the latter group of patients, whereas for the former group long-term azithromycin therapy may not be justifiable owing to its side effects and costs. One limitation of this therapeutic approach is that it is difficult (if not impossible) to predict the background rate of exacerbation for a given patient. Efforts are underway to develop simple and reasonably accurate clinical prediction tools using easily verifiable patient characteristics and traits, e.g. age, sex, number of exacerbations in the previous year and FEV1, that can be used clinically to estimate the future risk of exacerbations in a given patient [30].

\section{Event-based versus patient-based NNTs}

In the MACRO example, an event-based NNT was used to illustrate the importance of the background exacerbation rate of an individual patient. This approach may be reasonable given that patients can experience repeated exacerbation events in a given year. However, some endpoints are distinct, non-repeated events. Death would be one such example. The Towards a Revolution in COPD Health (TORCH) trial was powered on total mortality [31]. In this trial, 193 of the 1533 patients (12.6\%) in the fluticasone/salmeterol arm died, compared with 231 of the 1524 patients (15.2\%) in the placebo arm over 3 years of treatment. Thus, the NNT was $1 /(0.126-0.152)=38$. In other words, on average, the provision of fluticasone/salmeterol for 3 years would prevent one death among 38 patients who were treated with this therapy (compared with placebo). The TORCH trial also showed that fluticasone/salmeterol combination therapy was associated with an increased risk of pneumonia. The risk was $19.6 \%$ in the treatment arm compared with $12.3 \%$ in the placebo arm. The NNH was $1 /(0.196-0.123)=14$. Thus, there was one extra 
case of pneumonia for every 14 patients taking a fluticasone/salmeterol combination compared with placebo over 3 years.

A patient-based NNT (e.g. death or pneumonia NNT calculations for TORCH) is calculated by first dividing the number of patients who experience at least one event over the study period by the number of patients at risk for each of the study groups (i.e. absolute risk) and then taking the difference in the absolute risk between the study groups. For repeated events such as exacerbation, an event-based NNT can be calculated by first dividing the total number of exacerbations by the follow-up time for individuals in each of the study groups (thus generating absolute rates) and then taking the difference in the absolute rates between the study groups. Some have criticised this approach because the results are hard to interpret and, clinically, physicians treat patients and not events [32]. An alternative approach is to dichotomise the numerator of event-based NNTs by considering only the first exacerbation (or time to first exacerbation) and using the number of at-risk patients in the denominator (rather than person-time). This renders the following simple clinical interpretation: the number of patients needed to treat over a given unit of time (e.g. a year) to prevent at least one exacerbation over the study period [32]. In general, event-based NNTs are lower than patient-based NNTs. This is illustrated by the Informing the Pathway of COPD Treatment (IMPACT) trial for which the event-based NNT for exacerbations was approximately four, whereas the person-based NNT was approximately 34 comparing triple versus dual bronchodilator therapy over 1 year [33].

\section{NNTs and precision medicine}

In general, precision therapies are those that are associated with low NNTs. Indeed, the ideal precision medicine is one that has an NNT of one [34]. Even before the advent of the term "precision medicine", physicians were employing $\mathrm{N}$ of 1 trials (a randomised controlled crossover trial in a single patient designed to establish optimal treatment for that patient) to determine the efficacy of therapeutics. One notable example in COPD is theophylline [35]. Several decades ago, it was a widely held belief that theophyllines improved outcomes in COPD by inducing bronchodilation and providing anti-inflammatory effects. In 1999, MAHON et al. [35] performed a series of $\mathrm{N}$ of 1 trials to determine whether theophylline improved health status and exercise tolerance in patients with moderate to severe COPD. They found that theophylline had similar effects to placebo for these outcomes, a finding that was replicated in a recent large RCT [36] consisting of 1567 patients (compared with $\mathrm{n}=34$ for the study of MAHON et al. [35]). Given the enormous costs of bringing new drugs to the market, estimated to be $\$ 2.6$ billion per pill [37], in the era of precision medicine, $\mathrm{N}$ of 1 trials should be considered early on in the drug developmental process [34].

\section{Emerging predictive biomarkers of therapy Serum immunoglobulins}

Acute exacerbations account for a considerable share of the morbidity, mortality and economic burden of COPD, particularly for the approximate one-third of patients who experience frequent exacerbations $(\geqslant 2$ per year [38]). Early identification of patients with frequent exacerbations can help target therapies such as ICS, chronic azithromycin and roflumilast, all of which have been shown to reduce the frequency of these events. Given the close link between bacterial infections and COPD exacerbations [39], impairments in humoral immunity could explain the susceptibility to exacerbations in certain individuals. Indeed, immunoglobulin deficiency is fairly common in COPD patients, with one out of four patients with moderate to severe disease being IgG deficient [40]. Recent studies in the MACRO and Simvastatin for the Prevention of Exacerbations in COPD Exacerbations (STATCOPE) cohorts have demonstrated that serum IgG levels (readily measured in most clinical laboratories) can be used to identify patients at high risk for developing exacerbations (a prognostic biomarker) [40]. Specifically, IgG1 and IgG2 subclass deficiencies were most significantly associated with exacerbations and hospitalisations [41]. Whether Ig deficiency might also imply a possible therapeutic option for patients with frequent exacerbations, i.e. intravenous Ig (IVIG) replacement, remains to be determined. One small observational trial of eight COPD patients with Ig deficiencies receiving IVIG noted a reduction in the annual exacerbation rate from four to 0.5 [42], while another retrospective study of COPD patients who had received IVIG therapy reported a decrease in exacerbations from 4.7 to 0.6 per year [43]. Large RCTs have yet to be performed. Nonetheless, immunoglobulin measurement is a simple and accessible test that could rapidly identify the frequent exacerbator phenotype.

\section{Sputum microbiome}

With the advent of culture-independent methods of characterising microbial communities in the airways, sputum could be a good source of prognostic and even predictive biomarkers in COPD patients. Using bacterial 16s ribosomal RNA sequencing, a recent study profiled the sputum of 102 patients hospitalised 
with acute COPD exacerbations and found that patients who demonstrated infections with Staphylococcus species (which are pathogens in the lungs) had a sevenfold increase in the risk of 1-year mortality compared to hospitalised COPD patients without these organisms. Similarly, those who lost Veillonella species (which are commensals in the airway tract) in their sputum had a 13-fold increase in the risk of 1-year mortality. Importantly, those who demonstrated Staphylococcus but no Veillonella in their sputum had an 85-fold increase in the risk for 1-year mortality compared to those who retained Veillonella in their sputum [44]. Together, these data suggest that admission sputum samples could be used as prognostic biomarkers in acute COPD exacerbations and potentially guide therapeutic choices (e.g. antibiotics) at hospitalisation. Additional large studies will be required to validate this early observation.

\section{Prognostic imaging biomarkers}

In a recent review in this series, WASHKO and PARRAGa [45] discussed the use of CT and magnetic resonance imaging (MRI) biomarkers for precision medicine. Briefly, CT-based abnormalities such as emphysema (scored semi-quantitatively by radiologists) [46], parametric response mapping-related functional small airways disease [47] and reduced total airway count on CT scans [48] have all been shown to associate with a rapid decline in FEV1, especially in patients at risk of or with mild COPD. With further refinement, these imaging biomarkers have the potential to become prognostic biomarkers in mild or early COPD (as outlined previously by SORIANo et al. [49]) to predict individuals who are likely to experience rapid COPD progression and thus be candidates for early intervention and treatment for disease modification.

\section{Summary and conclusions}

Precision medicine is the future of COPD care and is enabled by the advent of biomarkers that can clearly identify subgroups of patients who will either benefit from novel therapies or experience only harm. Biomarkers are also required to gauge therapeutic responses during all phases of drug development to reduce failure rates and make drugs more affordable. To enable precision medicine, the benefits (as well as the harm) of therapeutics should be reported in both relative and absolute scales, which will enable calculation of metrics such as NNT and NNH. Finally, to reduce the cost of therapeutic trials (especially early on in development), drug companies should consider developing companion diagnostic tests to predict therapeutic and pharmacodynamic responses, and deploy $\mathrm{N}$ of 1 trials for therapies that target symptoms and modify risk (of exacerbations and/or disease progression) for committing to large-scale phase III trials.

Conflict of interest: J.M. Leung has nothing to disclose. M. Obeidat has nothing to disclose. M. Sadatsafavi has nothing to disclose. D.D. Sin reports funding for an investigator-initiated project to ascertain genomic determinants of COPD from Merck, honoraria for advisory meetings on COPD from Sanofi-Aventis and Regeneron, funding for clinical trials and investigator-initiated study of the effects of olodaterol on airway inflammation from Boehringer Ingelheim, funding for clinical trials and honoraria for speaking engagements and sitting on COPD advisory boards from AstraZeneca, and honoraria for speaking engagements and sitting on advisory boards from Novartis, outside the submitted work.

Support statement: D.D. Sin is a Tier 1 Canada Research Chair in COPD and holds the De Lazzari Family Chair at the Centre for Heart Lung Innovation (HLI). M. Sadatsafavi, M. Obeidat and J.M. Leung are Scholars with the Michael Smith Foundation for Health Research; M. Obeidat is a fellow of the Parker B Francis Foundation; M. Sadatsafavi receives salary support from The Canadian Institutes of Health Research.

\section{References}

1 Foreman KJ, Marquez N, Dolgert A, et al. Forecasting life expectancy, years of life lost, and all-cause and cause-specific mortality for 250 causes of death: reference and alternative scenarios for 2016-40 for 195 countries and territories. Lancet 2018; 392: 2052-2090.

2 The Tobacco Atlas. Prevalence. https://tobaccoatlas.org/topic/prevalence/ Date last accessed: December 24, 2018.

3 World Health Organization. Air Pollution. www.who.int/airpollution/en/ Date last accessed: December 24, 2018.

4 Miech R, Johnston L, O’Malley PM, et al. Adolescent vaping and nicotine use in 2017-2018 - U.S. National Estimates. N Engl J Med 2019; 380: 192-193.

5 Tan WC, Sin DD. What are the long-term effects of smoked marijuana on lung health? CMAJ 2018; 190: E1243-E1244.

6 Barnes PJ, Bonini S, Seeger W, et al. Barriers to new drug development in respiratory disease. Eur Respir J 2015; 45: 1197-1207.

7 Vogelmeier CF, Criner GJ, Martinez FJ, et al. Global Strategy for the Diagnosis, Management, and Prevention of Chronic Obstructive Lung Disease 2017 Report: GOLD executive summary. Eur Respir J 2017; 49: 1750214.

8 Sackett DL, Rosenberg WM, Gray JA, et al. Evidence based medicine: what it is and what it isn't. BMJ 1996; 312 : 71-72.

9 The Centre for Evidence-Based Medicine. www.cebm.net/ Date last accessed: December 24, 2018.

10 Rennard SI, Vestbo J. The many "small COPDs": COPD should be an orphan disease. Chest 2008; 134: 623-627.

11 Sin DD, Miravitlles M, Mannino DM, et al. What is asthma-COPD overlap syndrome? Towards a consensus definition from a round table discussion. Eur Respir J 2016; 48: 664-673. 
12 Collins FS, Varmus H. A new initiative on precision medicine. N Engl J Med 2015; 372: 793-795.

13 Jameson JL, Longo DL. Precision medicine - personalized, problematic, and promising. N Engl J Med 2015; 372: 2229-2234.

14 Biomarkers Definitions Working Group. Biomarkers and surrogate endpoints: preferred definitions and conceptual framework. Clin Pharmacol Ther 2001; 69: 89-95.

15 Agusti A, Bel E, Thomas M, et al. Treatable traits: toward precision medicine of chronic airway diseases. Eur Respir J 2016; 47: 410-419.

16 Kelloff GJ, Sigman CC. Cancer biomarkers: selecting the right drug for the right patient. Nat Rev Drug Discov 2012; 11: 201-214.

17 Fishman A, Martinez F, Naunheim K, et al.; National Emphysema Treatment Trial Research Group. A randomized trial comparing lung-volume-reduction surgery with medical therapy for severe emphysema. $N$ Engl $J$ Med 2003; 348: 2059-2073.

18 Cazzola M, Rogliani P, Calzetta L, et al. Triple therapy versus single and dual long-acting bronchodilator therapy in COPD: a systematic review and meta-analysis. Eur Respir J 2018; 52: 1801586.

19 Long term domiciliary oxygen therapy in chronic hypoxic cor pulmonale complicating chronic bronchitis and emphysema. Report of the Medical Research Council Working Party. Lancet 1981; 1: 681-686.

20 Long-Term Oxygen Treatment Trial Research Group, Albert RK, Au DH, et al. A randomized trial of long-term oxygen for COPD with moderate desaturation. N Engl J Med 2016; 375: 1617-1627.

21 Vogelmeier C, Hederer B, Glaab T, et al. Tiotropium versus salmeterol for the prevention of exacerbations of COPD. N Engl J Med 2011; 364: 1093-1103.

22 Criner GJ, Sue R, Wright S, et al. A multicenter randomized controlled trial of Zephyr endobronchial valve treatment in heterogeneous emphysema (LIBERATE). Am J Respir Crit Care Med 2018; 198: 1151-1164.

23 Churg A, Sin DD, Wright JL. Everything prevents emphysema: are animal models of cigarette smoke-induced chronic obstructive pulmonary disease any use? Am J Respir Cell Mol Biol 2011; 45: 1111-1115.

24 Cook D, Brown D, Alexander R, et al. Lessons learned from the fate of AstraZeneca's drug pipeline: a five-dimensional framework. Nat Rev Drug Discov 2014; 13: 419-431.

25 Rennard SI, Calverley PM, Goehring UM, et al. Reduction of exacerbations by the PDE4 inhibitor roflumilast - the importance of defining different subsets of patients with COPD. Respir Res 2011; 12: 18.

26 Maltby S, Gibson PG, Powell H, et al. Omalizumab treatment response in a population with severe allergic asthma and overlapping COPD. Chest 2017; 151: 78-89.

27 Chatellier G, Zapletal E, Lemaitre D, et al. The number needed to treat: a clinically useful nomogram in its proper context. BMJ 1996; 312: 426-429.

28 Albert RK, Connett J, Bailey WC, et al. Azithromycin for prevention of exacerbations of COPD. N Engl J Med 2011; 365: 689-698.

29 Sadatsafavi M, Sin DD, Zafari Z, et al. The association between rate and severity of exacerbations in chronic obstructive pulmonary disease: an application of a joint frailty-logistic model. Am J Epidemiol 2016; 184: 681-689.

30 Sadatsafavi M, Gustafson P, Zafari Z, et al. Relative impact characteristic curve: a graphical tool to visualize and quantify the clinical utility and population-level consequences of implementing markers. Ann Epidemiol 2018; 28: 717-723.

31 Calverley PM, Anderson JA, Celli B, et al. Salmeterol and fluticasone propionate and survival in chronic obstructive pulmonary disease. N Engl J Med 2007; 356: 775-789.

32 Suissa S. Number needed to treat: enigmatic results for exacerbations in COPD. Eur Respir J 2015; 45: 875-878.

33 Lipson DA, Barnhart F, Brealey N, et al. Once-daily single-inhaler triple versus dual therapy in patients with COPD. N Engl J Med 2018; 378: 1671-1680.

34 Schork NJ. Personalized medicine: time for one-person trials. Nature 2015; 520: 609-611.

35 Mahon JL, Laupacis A, Hodder RV, et al. Theophylline for irreversible chronic airflow limitation: a randomized study comparing $\mathrm{n}$ of 1 trials to standard practice. Chest 1999; 115: 38-48.

36 Devereux G, Cotton S, Fielding S, et al. Effect of theophylline as adjunct to inhaled corticosteroids on exacerbations in patients with COPD: a randomized clinical trial. JAMA 2018; 320: 1548-1559.

37 Avorn J. The \$2.6 billion pill - methodologic and policy considerations. N Engl J Med 2015; 372: $1877-1879$.

38 Hurst JR, Vestbo J, Anzueto A, et al. Susceptibility to exacerbation in chronic obstructive pulmonary disease. N Engl J Med 2010; 363: 1128-1138.

39 Monso E, Ruiz J, Rosell A, et al. Bacterial infection in chronic obstructive pulmonary disease. A study of stable and exacerbated outpatients using the protected specimen brush. Am J Respir Crit Care Med 1995; 152:1316-1320.

40 Leitao Filho FS, Won Ra S, Mattman A, et al. Serum IgG and risk of exacerbations and hospitalizations in chronic obstructive pulmonary disease. J Allergy Clin Immunol 2017; 140:1164-1167.

41 Leitao Filho FS, Ra SW, Mattman A, et al. Serum IgG subclass levels and risk of exacerbations and hospitalizations in patients with COPD. Respir Res 2018; 19: 30.

42 McCullagh BN, Comellas AP, Ballas ZK, et al. Antibody deficiency in patients with frequent exacerbations of chronic obstructive pulmonary disease (COPD). PLoS One 2017; 12: e0172437.

43 Cowan J, Gaudet L, Mulpuru S, et al. A retrospective longitudinal within-subject risk interval analysis of immunoglobulin treatment for recurrent acute exacerbation of chronic obstructive pulmonary disease. PLoS One 2015; 10: e0142205.

44 Leitao Filho FS, Alotaibi NM, Ngan D, et al. Sputum microbiome is associated with 1-year mortality following COPD hospitalizations. Am J Respir Crit Care Med 2018; in press [https://doi.org/10.1164/rccm.201806-1135OC].

45 Washko GR, Parraga G. COPD biomarkers and phenotypes: opportunities for better outcomes with precision imaging. Eur Respir J 2018; 52: 1801570.

46 Yuan R, Hogg JC, Pare PD, et al. Prediction of the rate of decline in FEV1 in smokers using quantitative computed tomography. Thorax 2009; 64: 944-949.

47 Bhatt SP, Soler X, Wang X, et al. Association between functional small airway disease and FEV1 decline in chronic obstructive pulmonary disease. Am J Respir Crit Care Med 2016; 194: 178-184.

48 Kirby M, Tanabe N, Tan WC, et al. Total airway count on computed tomography and the risk of chronic obstructive pulmonary disease progression. Findings from a population-based study. Am J Respir Crit Care Med 2018; 197: 56-65. 
49 Soriano JB, Polverino F, Cosio BG. What is early COPD and why is it important? Eur Respir J 2018; $52: 1801448$.

50 van Gool AJ, Bietrix F, Caldenhoven E, et al. Bridging the translational innovation gap through good biomarker practice. Nat Rev Drug Discov 2017; 16: 587-588.

51 Freedman LP, Cockburn IM, Simcoe TS. The economics of reproducibility in preclinical research. PLoS Biol 2015; 13: e1002165.

52 Wilkinson MD, Dumontier M, Aalbersberg IJ, et al. The FAIR Guiding Principles for scientific data management and stewardship. Sci Data 2016; 3: 160018. 